\title{
AMCP Partnership Forum: Driving New Advances in Dyslipidemia Management
}

\begin{abstract}
SUMMARY
Proprotein convertase subtilisin kexin 9 (PCSK9) inhibitors are specialty medications approved for the treatment of dyslipidemia. Because dyslipidemia is a prevalent and chronic condition, the costs associated with PCSK9 inhibitors must be carefully weighed against the benefits that they provide. However, the long-term clinical benefits of these agents are not yet fully defined, creating a complex situation for third-party payers who must determine how to allocate limited resources.

To engage national stakeholders in a discussion of how to wisely use PCSK9 inhibitors in health care, the Academy of Managed Care Pharmacy organized a forum in Washington, DC, on September 16, 2015. The forum began with presentations reviewing the current environment for dyslipidemia treatment. Following these presentations, participants engaged in discussions regarding how to best select patients for treatment based on current treatment guidelines, how managed care pharmacy can best manage high-cost specialty products that may be indicated for large numbers of patients, and how managed care organizations can use real-world evidence to assess the impact of evolving treatment guidelines and options.

Participants called for the development of a national database to gather more meaningful data regarding the impact of PCSK9 inhibitors on clinical outcomes. Such a database would be invaluable for determining the real-world impact of specialty medications and would support the development of sensible third-party payer benefit structures, coverage decisions, medical policies, and risk-sharing contracts. In the meantime, participants recommended enhanced collaboration among stakeholders for improved information sharing, increased collection and evaluation of real-world evidence, use of available costeffectiveness analyses, and the implementation of strategies that optimize the use of evidence-based therapies.
\end{abstract}

J Manag Care Spec Pharm. 2016;22(4):426-33

Copyright $\odot 2016$, Academy of Managed Care Pharmacy. All rights reserved.

$\mathrm{I}$ n 2015, the U.S. Food and Drug Administration (FDA) approved 2 drugs in a new class of specialty medications known as proprotein convertase subtilisin kexin 9 (PCSK9) inhibitors. These specialty medications are approved for the treatment of patients with dyslipidemia, which is associated with the development of atherosclerotic cardiovascular disease (ASCVD) and includes myocardial infarction and stroke. Approximately one-third of American adults have ASCVD, and it is the most common cause of death in the United States. ${ }^{1}$

Randomized clinical trials have demonstrated that lowering low-density lipoprotein cholesterol (LDL-C) with statin therapy reduces the risk of myocardial infarction, stroke, and death in patients with cardiovascular disease. ${ }^{2-4}$ Based on this evidence, current national guidelines recommend statin therapy as the primary pharmacologic treatment of elevated LDL-C. However, there remains an open debate about optimal LDL-C level targets in dyslipidemia management, since several drugs have been shown to lower LDL-C in randomized trials without demonstrating statistically significant reductions in cardiovascular disease events. PCSK9 inhibitors were approved based on their efficacy for lowering LDL-C. Ongoing studies are evaluating their impact on ASCVD events and long-term safety.

While the new PCSK9 inhibitors show promise for improving patient health, they join a growing list of high-cost specialty medications that pose financial challenges for health care payers. This trend toward introduction of expensive specialty medications raises concern about the sustainability and affordability of health care. Sustainability issues are particularly pronounced for PCSK9 inhibitors because of their potential for widespread and long-term use.

At the marketed list price of approximately $\$ 14,000$ annually per patient (as of 2015), PCSK9 inhibitors have the potential to have a significant and long-term impact on the financial stability of managed care organizations. ${ }^{5}$ While the price may decrease over time as new products enter the market and competition increases, the availability of a specialty medication that is indicated for long-term therapy in a broad range of patients requires appropriate management strategies to ensure that these therapies are targeted to patients who will derive the greatest benefit.

As more high-cost specialty medications enter the market, the health care system must prepare a rational response to ensure that the benefits of emerging therapies for society are realized, while also maximizing value of less expensive therapies. Collaborations among stakeholders to gather and analyze relevant data will be crucial for designing third-party payer policies that optimize the value of emerging therapies.

\section{Looking at the Landscape}

To engage national stakeholders in a discussion of how to wisely use PCSK9 inhibitors in health care, the Academy of Managed Care Pharmacy (AMCP) organized a Partnership Forum entitled "Driving New Advances in Dyslipidemia Management" in Washington, DC, on September 16, 2015. This forum allowed participants the opportunity to collaborate, gain insights about experiences managing specialty medications, and share perspectives for guiding drug therapy management decisions. Stakeholder participants included representatives from academia, research, insurers, manufacturers, industry, and managed care. The event was supported by Lilly USA, MedImpact Healthcare Systems, Merck \& Co., PerformRx, Pfizer, and Sanofi. 
The forum began with presentations on dyslipidemia treatment guidelines, a review of the research pipeline for medications used in the treatment of dyslipidemia, management solutions to improve patient and treatment outcomes, and a discussion of the cost-effectiveness of therapy. Following these presentations, participants engaged in several discussions regarding pressing issues for managed care organizations, including (a) how to best select patients for treatment based on current guidelines for the treatment of dyslipidemia, (b) how managed care pharmacy can best manage high-cost specialty products that may be indicated for large numbers of patients, and (c) how managed care organizations can use real-world evidence to assess the impact of evolving treatment guidelines and options.

\section{Guidelines for the Treatment of Dyslipidemia}

According to data from the National Health and Nutrition Examination Survey (2003-2006), approximately 27\% of adults in the United States have elevated LDL-C. ${ }^{6}$ The use of pharmacotherapy to manage dyslipidemia is widespread and has increased from 3.4\% of the population in 1988-1994 to 15.5\% in 2007-2010. This increase was accompanied by growth in the number of available treatments, including high potency statins, and has been associated with improvements in mean lipid values over the same time period. ${ }^{7}$

Joseph J. Saseen, professor and vice chair at the University of Colorado Skaggs School of Pharmacy, provided an overview of dyslipidemia treatment guidelines. The goal of dyslipidemia treatment is to reduce the risk of experiencing ASCVD events (e.g., myocardial infarction and stroke). However, LDL-C levels are often used as an intermediate endpoint to assess the efficacy of treatment. As shown by a meta-analysis of 14 trials involving 90,056 participants, a $1 \mathrm{mmol} / \mathrm{L}(38.6 \mathrm{mg} / \mathrm{dL})$ reduction in LDL-C with statins is associated with a $22 \%$ reduction in cardiovascular events. ${ }^{2}$

For decades, treatment of dyslipidemia in the United States was driven by guidelines from the National Cholesterol Education Program (NCEP). These guidelines focused on evaluating individual patient risk for coronary heart disease (CHD) and calculating 10-year CHD risk based on the Framingham risk calculator for certain primary prevention patients and then matching intensity of therapy and recommending LDL-C treatment goals based on patient risk. ${ }^{8}$ The NCEP guidelines recommended using a combination of statins along with other nonstatin medications as needed to reach LDL-C goals.

The 2013 American College of Cardiology and American Heart Association (ACC/AHA) guidelines recommend a different approach to the treatment of dyslipidemia. These guidelines use the Pooled Cohort Equations to assess risk in many primary prevention patients and identify groups of patients expected to benefit from statin therapy. These guidelines also incorporated new terminology and refer to ASCVD rather than previously used terms such as CVD or CHD.

While the Framingham risk calculator only considers risk of myocardial infarction, the Pooled Cohort Equations consider risk for stroke and myocardial infarction. Recent evidence indicates that the Pooled Cohort Equations more accurately identifies high-risk patients than the Framingham risk calculator. ${ }^{9}$

Treatment recommendations in the current ACC/AHA guidelines are based on available clinical trial evidence and focused on clinical trials that reported rates of ASCVD outcomes. The available evidence base of randomized controlled trials for the treatment of dyslipidemia primarily uses fixed doses of cholesterol-lowering medications (mostly statins) to reduce ASCVD risk. There were no direct data based on clinical trials that support titrating patients to a specific LDL-C treatment goal. Therefore, even though lower LDL-C levels are associated with lower ASCVD risk, the guidelines do not recommend specific LDL-C treatment goals. ${ }^{10}$ Instead, the guidelines recommend either moderate- or high-intensity statin therapy for 4 groups of patients. Moderate-intensity statin therapy is an option if high-intensity statin therapy is contraindicated or if patients are at increased risk for statinassociated adverse effects. ${ }^{10}$

The ACC/AHA guidelines make few recommendations for when nonstatin therapies should be used, noting that there were no available data showing that adding a nonstatin drug to a statin produced a further reduction in the rate of ASCVD events compared with a statin alone. The guidelines did note that for patients with untreated LDL-C of $\geq 190 \mathrm{mg} / \mathrm{dL}$, it is reasonable to intensify statin therapy to achieve at least a 50\% reduction in LDL-C and that addition of a nonstatin drug may be considered to further lower LDL-C. In addition, for individuals who are candidates for statin therapy but are completely statin intolerant, it is reasonable to use nonstatin drugs that have been shown to reduce ASCVD events if benefits outweigh the potential for adverse effects. ${ }^{10}$

The IMPROVE-IT trial was released after the ACC/AHA guidelines were published. This trial of more than 18,000 high-risk patients found that, in addition to supplemental LDL-C lowering, the addition of ezetimibe to a statin further reduced the risk of ASCVD compared with a statin alone. ${ }^{11}$ This trial provides evidence supporting the concept that adding a nonstatin to a statin can further lower LDL-C and provide additional ASCVD risk reduction.

Another set of guidelines was released by the National Lipid Association (NLA) in 2014. While the NLA also identified statins as the foundation of dyslipidemia pharmacotherapy, NLA retained LDL-C treatment targets as goals of therapy. These guidelines continue to call for adjusting the intensity of pharmacotherapy based on the patient's absolute risk to achieve specific LDL-C goal values. It is important to note that while ACC/AHA provided recommendations that were strictly based 
on clinical evidence, the NLA included recommendations based on expert opinion. ${ }^{12}$

Neither the ACC/AHA nor the NLA guidelines discuss the use of PCSK9 therapies because these agents were not approved at the time of their publication. Some authors have argued that the introduction of these specialty therapies should precipitate a return to guidelines based on treatment goals to support the judicious use of these new therapies. ${ }^{13}$

\section{Emerging Therapies for Dyslipidemia}

Seth S. Martin, assistant professor of medicine at Johns Hopkins University School of Medicine, described recent innovations in medications for the management of dyslipidemia, focusing on PCSK9 inhibitors and cholesterol ester transfer protein (CETP) inhibitors.

The inhibition of PCSK9 activity enhances the clearance of LDL-C from the blood and adds to the efficacy of statin therapy for lowering LDL-C. ${ }^{14}$ PCSK9 inhibitors, including evolocumab, alirocumab, and bococizumab, are monoclonal antibodies that are injected subcutaneously. Evolocumab and alirocumab have been approved by the FDA and are indicated as an adjunct to diet and maximally tolerated statin therapy for the treatment of adults with heterozygous familial hypercholesterolemia $(\mathrm{HeFH})$ or clinical ASCVD, who require additional lowering of LDL-C. Evolocumab is also approved for treatment of homozygous familial hypercholesterolemia (HoFH). Bococizumab is another PCSK9 inhibitor that is in late-stage clinical development.

Available evidence demonstrates that PCSK9 inhibitors lower LDL-C approximately 50\%-60\% compared with standard therapy among patients who are already taking statins or who cannot tolerate them. ${ }^{15,16}$ Whether these lower cholesterol levels will translate into lower ASCVD rates is currently being investigated in long-term trials. Some participants reported that prescribers are currently taking a cautious approach to the use of PCSK9 inhibitors. In current clinical practice, their use has been generally limited to patients with familial hypercholesterolemia $(\mathrm{FH})$. It remains to be seen whether clinicians will prescribe these medications to a wider pool of patients as they gain more familiarity with them.

There are some emerging data that suggest PCSK9 inhibitors do have benefits for the reduction of ASCVD events. For example, 2 open-label trials comprising 4,465 patients found that the rate of cardiovascular events at 1 year was reduced from $2.18 \%$ in patients receiving standard therapy to $0.95 \%$ in patients also receiving evolocumab $(P=0.003){ }^{16} \mathrm{~A}$ metaanalysis of 24 phase II and phase III clinical trials of PCSK9 inhibitors that comprised over 10,000 patients found that PCSK9 therapy reduced all-cause mortality $(P=0.015)$ and myocardial infarction $(P=0.03)$ and trended toward a reduction in cardiovascular mortality $(P=0.084)$. PCSK9 therapy did not increase rates of serious adverse events. ${ }^{17}$ However, prospective randomized controlled clinical trials are necessary to provide more definitive evidence. Ongoing ASCVD outcomes trials include the FOURIER trial of evolocumab, the ODYSSEY Outcomes trial of alirocumab, and the SPIRE-2 trial of bococizumab..$^{18-20}$

CETP inhibitors are another class of agents under investigation for the treatment of ASCVD. Although they have been shown to raise high-density lipoprotein cholesterol and lower LDL-C, clinical trials have yielded mixed results. The CETP inhibitor torcetrapib failed phase II clinical trials because of excess deaths attributed to off-target effects, and dalcetrapib also failed during phase III trials because of lack of efficacy in reducing ASCVD. Recently, the clinical trial program for another CETP inhibitor, evacetrapib, has been halted because of lack of efficacy in an interim analysis. ${ }^{21}$ One additional CETP inhibitors, anacetrapib, notable for stronger LDL-C lowering, is currently in phase III clinical trials. Once more data are available, the potential role of this drug class in dyslipidemia therapy will be better determined. ${ }^{22}$

\section{Managing Medication Costs in an Evolving Health Care System}

Patty Kumbera, a consultant at Kumbera Solutions, discussed management solutions to improve quality outcomes and affordability. Historically, payers managed medical costs separately from pharmacy costs. This fragmented structure can hinder the implementation of strategies that reduce total health care costs. Greater integration by third-party payers can support the use of expensive but cost-effective medications. The increased focus on pay for performance (P4P), which has emerged since the implementation of the Affordable Care Act, is an important factor driving payer integration, particularly for specialty pharmacy. However, silos still exist in some areas and remain a barrier to cost-effective use of medications. ${ }^{23}$

Payment systems are being realigned to ensure that resources are being used wisely and that stakeholders are considering quality of care as well as total health care costs. As a result, health care payers increasingly have incentives to improve the quality of care and increasingly rely on quality measures. The movement toward P4Ps and other value-based payment structures may support these developments and enhanced provider consideration of cost-effectiveness.

Many existing quality measures that focus on dyslipidemia were developed before the release of the ACC/AHA guidelines and are based on achieving LDL-C goals that were defined in the NCEP guidelines. ${ }^{8,10,24,25}$ Some measures have been modified to reflect new guidelines but others continue to consider specific lipid levels as treatment goals. 
However, even with current guidelines and quality measures that support statin therapy, patient adherence to medication therapy remains a key barrier to achieving optimal patient outcomes. Greater involvement of prescribers and pharmacists in the process can help to support patient adherence to therapy, ensure that statin use is optimized before considering additional therapies, and potentially influence provider behavior to consider the financial impact of treatment decisions.

\section{Assessing the Value of Costly Therapies}

Finally, James D. Chambers, assistant professor at the Center for the Evaluation of Value and Risk in Health at Tufts Medical Center, discussed the value of specialty medications and use of real-world evidence. He explored the health impacts of specialty medications, measured in quality-adjusted life year (QALY) gains, and he identified important distinctions between randomized controlled trials and real-world evidence.

Chambers presented an analysis of published estimates of QALYs compared with medication costs and health care resource use among 58 specialty drugs and 44 traditional drugs that were approved by the FDA between 1999 and 2011. ${ }^{26}$ This analysis found that, compared with preexisting treatments, new-to-market specialty drugs provided greater gains in QALYs compared with new-to-market traditional drugs, and the 2 classes of drugs had comparable cost-effectiveness. This analysis concluded that, although specialty drugs may cost more than traditional drugs, they also may provide greater benefits and therefore offer value for the health care system. ${ }^{26}$

Nevertheless, the cost burden of specialty medications is substantial and must be carefully managed. The Institute for Clinical and Economic Review (ICER) released a revised draft report on the effectiveness, value, and pricing benchmarks for PCKS9 inhibitors. This report estimated that approximately 2.6 million Americans would receive treatment with PCSK9 inhibitors for 1 or more years between 2015 and 2020. This analysis determined that the potential budget impact would be nearly $\$ 19$ billion per year to provide PCSK9 inhibitors for 25\% of the eligible patient population. ${ }^{27}$

However, it is important to realize that available data are from a specific, narrowly defined population of patients who participated in regimented study protocols. There is uncertainty regarding the value of these agents for patients with various clinical characteristics during real-world use of medications with less carefully controlled conditions. Therefore, results from trials that are used as the basis for approvals from the FDA do not necessarily translate into results in the real world.

Data from large observational studies are necessary to assess real-world treatment effects of PCSK9 inhibitors in patients with a wider range of clinical characteristics. Given the dearth of long-term data regarding the value of PCSK9 inhibitors for reducing ASCVD outcomes, the results of ongoing outcomes trials with these agents will be crucial.

\section{Strategies for Moving Forward}

Forum participants engaged in interactive discussions to explore issues related to appropriate management of dyslipidemia, as well as global strategies to encourage appropriate use of highcost specialty medications and ensure that the health insurance marketplace remains sustainable.

\section{Guidelines and Quality}

Participants explored strategies that managed care organizations can use to promote quality of care for patients with dyslipidemia. The conversation addressed the need for guidelines and quality measures to be aligned and for payers to have access to current real-world data. Furthermore, they emphasized that proven therapies (e.g., statins) should be fully utilized before patients try medications with a more limited evidence base, such as the PCSK9 inhibitors, and that prior authorization requirements could support this goal.

According to participants, $\mathrm{P} 4 \mathrm{P}$ incentives are proving useful for improving quality of care and patient outcomes. Such incentives generally require the use of objective measures of quality to assess performance. However, the ACC/AHA guidelines lack specific lipid treatment goals that could provide quantifiable measures on which to base the development of prior authorization requirements and risk-sharing contracts. Many participants indicated that a return to lipid goalbased guidelines would be more useful for designing quality measures and prior authorization requirements for managing dyslipidemia.

Although the ACC/AHA guidelines were developed before the approval of PCSK9 inhibitors, they do include some criteria that could be used to develop prior authorization or P4P criteria. The guidelines provide guidance for the use of nonstatin medications for high-risk patients who have a less-than-anticipated response to statins, who are unable to tolerate a recommended intensity of a statin, or who are statin intolerant.

PCSK9 inhibitors are approved as an adjunct to diet and maximally tolerated statin therapy for high-risk patients with ASCVD and FH patients. However, identifying patients who cannot tolerate statins is difficult. As a result of the imprecise nature of statin intolerance, managed care organizations have implemented numerous criteria to validate its presence before granting prior authorization for PCSK9 therapy. Some organizations require patients to fail a therapy trial with 1 statin, while others require that patients fail to tolerate multiple statins. A consistent evidence-based definition of, or criteria to evaluate, statin intolerance could better support consistency among clinicians and payers around treatment and coverage decisions.

In the absence of updated guidelines, clinical trial data may be used to better define appropriate uses of PCSK9 inhibitors 
and associated quality measures. However, current efficacy data regarding the PCSK9 inhibitors are based primarily on LDL-C levels. Clinical trials regarding efficacy for reducing rates of ASCVD events and establishing long-term safety are currently underway, but in the meantime, payers must rely on surrogate endpoints (e.g., LDL-C levels) to make decisions about structuring benefits and are justifiably reluctant to allow unrestricted use.

Participants also recommended implementing strategies to ensure that providers are optimizing the use of statins before prescribing a PCSK9 inhibitor. Patient adherence is particularly difficult for dyslipidemia because there are usually no symptoms until an ASCVD event occurs. Therefore, participants noted, the patient may be less likely to be willing to tolerate any adverse effects associated with the medication. Patient adherence to statin therapy should be assessed and fully supported before adding nonstatin therapies.

Prior authorization is often used as a mechanism to ensure that expensive therapies are limited to patients most likely to derive benefit from them. In lieu of presented or published long-term outcomes on ASCVD events, potential criteria for prior authorization could include a patient's LDL-C while receiving maximally tolerated statins with or without other lipid-lowering therapy, history of ASCVD risk or event, diagnosis or physician attestation of $\mathrm{FH}$, documentation of intolerance to multiple statins (with trial duration and dose), and demonstration of satisfactory adherence to the current dyslipidemia treatment regimen.

\section{Evolving Treatment Strategies}

Developments that affect dyslipidemia management since the publication of the ACC/AHA treatment guidelines include the approval of PCSK9 inhibitors, the release of IMPROVE-IT trial results, and the potential for future medications (e.g., CETP inhibitors) to enter the market. Participants explored the impact of these developments on the treatment of dyslipidemia for managed care organizations from an affordability, appropriate use, and access standpoint.

The conversation explored better planning and collaboration among stakeholders to address new drugs before they are launched in the market. Because payers generally develop their budgets and benefits structures years in advance, the approval of a new expensive treatment that may be used by a large segment of the population can be difficult to absorb, particularly when there is little prior notice for payers. Participants noted that there is a lack of prospective information about the pricing for new medications and that once the medications are approved payers must rapidly implement effective management strategies. Earlier communication about new medication pricing would allow for better budget preparation and could improve collaboration across key stakeholders.
Participants suggested that analyses that examine the costeffectiveness of a drug, such as those that are performed by ICER, could assist with establishing the price of specialty products. They suggested that payers could support policies that base pricing on proven cost-effectiveness.

Participants noted that AMCP could continue to facilitate discussions across stakeholder groups to enhance the interpretation, implementation, and management of advances in dyslipidemia therapy. It was noted that provider groups and patient advocacy groups should be included as key stakeholders for future discussions. This type of collaboration could help define best practices in the absence of up-to-date guidelines. These collaborations may also incorporate the use of real-world data to inform coverage decisions and increase confidence in those decisions.

Other recommended collaborations included designing clinical trials to answer questions important to managed care organizations. Currently, clinical studies are designed to demonstrate safety and efficacy; these data may not always provide the information necessary to determine the value of a new product in specific patient populations or support clinical policy decisions. Payers, the Centers for Medicare \& Medicaid Services (CMS), the FDA, and the pharmaceutical industry could collaborate regarding study designs to support development of clinical trials that will provide data to support payer decision making.

\section{Gathering Data to Support Rational Decision Making}

Prescribers often are hesitant to discuss medication cost issues with patients given the complexity of various insurance benefit designs and a desire to avoid the appearance of basing treatment decisions on an individual's ability to pay for a medication. Several participants recommended that AMCP could collaborate with a medical association to develop tools to support provider-patient dialogue about the high cost of specialty medications.

In the meantime, the burden generally rests on payers to be good stewards of limited health care dollars. Given that treatment with a PCSK9 inhibitor is expensive and could potentially be given to a single patient for decades, it is wise for payers to base coverage criteria on the clinical evidence and to explore risk-sharing agreements with key stakeholders who influence the use of medications (e.g., provider groups) and determine the price of the medication (e.g., pharmaceutical manufacturers). The cost savings associated with reduced ASCVD events should be weighed against the cost of the medication to determine the added value it provides compared with previously available therapies. These data should be combined with additional data from cost-effectiveness analysis to inform the design of coverage decisions and risk-sharing contracts. 
Proposed Database Development. There is a need to have access to real-time medication utilization information across the whole treatment population so that payers can evaluate data and make recommendations in a timely manner. If available data demonstrate that a new therapy significantly improves clinical outcomes, then payers will have clinical justification to make the therapy available to the appropriate patient populations as soon as possible. Real-world evidence can also be used to guide the development of coverage criteria and risksharing contracting arrangements.

Several participants explored options for managed care organizations to collect and analyze data to measure the impact of new therapies and drive effective decision making. Participants stressed the need for meaningful, real-time, and real-world actionable data, and some called for the development of a national database that would address stakeholder needs. Collecting such data is challenging but could potentially be done through a database similar to the types of registries that have been implemented for other medications. Ideally, registries should be established on a national level so that outcomes for patients who switch among plans can be included in the dataset.

The database for tracking the value of specialty medications should allow for comparisons, organizational tracking, and independent analyses using real-time deidentified data. Examples of existing databases upon which a database or registry could be modeled include the Medicare Medic, payers' claims databases, state-level prescription drug monitoring programs for opioids, and the Medicare Part D Overutilization Monitoring System.

Key information to include in the database would be patient demographic data, prescriptions, diagnosis and procedure codes, and laboratory data. Locating such data in a patient registry could facilitate stakeholder access to the meaningful, real-time, and real-world evidence needed to make appropriate updates to medical and pharmacy policies or coverage criteria.

Participants identified stakeholders who should be involved in developing the database, including payers, providers, pharmacists, associations, pharmacy benefit managers, pharmaceutical manufacturers, health economists, technology companies, and federal groups such as the FDA and CMS. Ideally, the database would be led by an independent group. Several participants proposed that managed care organizations or AMCP could act as a central organization for developing partnerships and seeking the consensus needed to develop and maintain such a database.

\section{Conclusions}

The availability of breakthrough drugs that provide value for large numbers of patients but pose affordability challenges must be managed to maintain the sustainability of the health care system. While evidence-based coverage limits are being put in place for many specialty medications, the system must maintain incentives for pharmaceutical manufacturers to develop and bring products to market that benefit society. Therefore, there is value in creating a careful balance in which stakeholders collaborate to ensure appropriate use of medications.

In the treatment of dyslipidemia, statins have been shown to improve ASCVD outcomes, and ezetimibe is the only medication that has been shown to provide further ASCVD lowering when added to a statin. Other available lipid-lowering therapies have been shown to improve LDL-C levels but lack evidence demonstrating their impact on ASCVD outcomes. PCSK9 inhibitors lower LDL-C dramatically when added to a statin, and preliminary outcomes regarding ASCVD events are encouraging, but trials are ongoing to more definitively determine their long-term impact.

Guidelines and quality measures are often used as the basis for structuring benefits, making coverage decisions, and establishing risk-sharing contracts. Moving forward, stakeholders require more meaningful data regarding the impact of PCSK9 inhibitors on clinical outcomes. This will allow for rational decision making rather than relying on data from surrogate endpoints (e.g., LDL-C levels) for coverage decision making. There is a need for the development of a national database that will provide insights into the real-world impact of specialty medications in real time. Such a database would be invaluable for identifying patients most likely to benefit from therapy and would support sensible benefit structures, coverage decisions, medical policies, and risksharing contracts.

Efforts to collaborate with physicians on their educational needs regarding managed care best practices, to gain insights from provider and patient groups on best practices for the management of dyslipidemia, and to promote population care management principles in the design of clinical trials would be worthwhile endeavors for AMCP. In the meantime, enhanced collaboration among stakeholders, increased collection and evaluation of real-world evidence, use of available costeffectiveness analyses, and the promotion of strategies that optimize the use of evidence-based therapies will be crucial for the treatment of dyslipidemia and for the sustainability of the health care system. 


\section{Forum Participants}

MONICA ADAMS, PharmD, Clinical Training Specialist, Sinfonia Rx; AMBARISH J. AMBEGAONKAR, PhD, Senior Director Team Leader, Global Health and Value, Pfizer; GARY BESINQUE, PharmD, FCSHP, Pharmacist Evidence Analyst and Strategist, Kaiser Permanente Medical Care Plan; KRISTINE K. BORDENAVE, MD, FACP, Lead Medical Director, Humana; KATHRYN R. BROWN, PharmD, MHA, Director Pharmacy Services, Premera Blue Cross; LISA CASHMAN, PharmD, Director, Clinical Formulary, MedImpact Healthcare Systems; JAMES D. CHAMBERS, MPharm, MSC, PhD (presenter), Assistant Professor, Center for the Evaluation of Value and Risk in Health, Tufts Medical Center; JUDY CRESPI-LOFTON, MS, Medical Writer \& Consultant, JCL Communications; JESSICA DAW, PharmD, MBA, Director, Clinical Pharmacy, UPMC Health Plan; JEFFREY DZIEWECZYNASKI, RPh, MS, Formulary Development/Rebate Contract Management, Blue Cross Blue Shield Association; JAMES R. HOPSICKER, RPh, MBA, Vice President, Pharmacy Programs, MVP Health Care; PATTY A. KUMBERA, RPh, (presenter), Consultant, Kumbera Solutions; ABBEY LEFRANCOIS, PharmD, Director, Clinical Solutions, Express Scripts; GREG LOW, RPh, PhD, Director, MGPO Quality and Utilization Program, Massachusetts General Hospital; STEVEN LUCIO, PharmD, BCPS, Senior Director, Clinical Pharmacy Solutions, Novation; DANI MARKUS, PharmD, MBA, Director of Clinical Services, OutcomesMTM; SETH S. MARTIN, MD, MHS (presenter), Assistant Professor of Medicine, Johns Hopkins University School of Medicine; CRAIG MATTSON, MS, MBA, RPh, Senior Director, Formulary Development, Prime Therapeutics; SAMIR MISTRY, PharmD, Senior Director, Specialty Products, CVS/ Caremark; CLIFF MOLIFE, PhD, Health Outcomes Research Scientist, Lilly USA; JOSEPH J. SASEEN, PharmD, BCPS, BCACP (presenter), Professor and Vice Chair, University of Colorado Skaggs School of Pharmacy; SHAILI SHAH, PharmD, Clinical Pharmacist, Formulary Management \& DUR, PerformRx, JANN B. SKELTON, BPharm, MBA, President, Silver Pennies Consulting; JEFF TAYLOR, RPh, MS, Pharmacy Director, Aetna.

AMCP STAFF: CHARLIE DRAGOVICH, Senior Director, Strategic Alliances and Business Development; SARAH ENFIEDJIAN, Program Coordinator; DENISE HEDDLESTEN, Program Coordinator; SUSAN OH, PharmD, MS, Assistant Director of Pharmacy Affairs; TERRY RICHARDSON, PharmD, BCACP, Director of Product Development; SOUMI SAHA, PharmD, JD, Assistant Director of Pharmacy and Regulatory Affairs; PUNEET SINGH, PharmD, Manager of Education; RUBY SINGH, PharmD, BCPS, Vice President of Education and Training.

CORRESPONDENCE: Terry Richardson, PharmD, BCACP, Director of Product Development, Academy of Managed Care Pharmacy, 100 N. Pitt St., Ste. 400, Alexandria, VA 22314.

E-mail: trichardson@amcp.org.

\section{ACKNOWLEDGMENTS}

This proceedings document was written by Judy Crespi-Lofton, MS, Medical Writer \& Consultant, JCL Communications.

\section{REFERENCES}

1. Mozaffarian D, Benjamin EJ, Go AS, et al. Heart disease and stroke statistics-2015 update: a report from the American Heart Association. Circulation. 2015;131(4):e29-322.

2. Cholesterol Treatment Trialists' (CTT) Collaboration, Baigent C, Blackwell L, et al. Efficacy and safety of more intensive lowering of LDL cholesterol: a meta-analysis of data from 170,000 participants in 26 randomised trials. Lancet. 2010;376(9753):1670-81.

3. Cholesterol Treatment Trialists' (CTT) Collaboration, Fulcher J, O'Connell R, et al. Efficacy and safety of LDL-lowering therapy among men and women: meta-analysis of individual data from 174,000 participants in 27 randomised trials. Lancet. 2015;385(9976):1397-405.

4. Taylor F, Huffman MD, Macedo AF, et al. Statins for the primary prevention of cardiovascular disease. Cochrane Database Syst Rev. 2013;1:CD004816.

5. Schulman KA, Balu S, Reed SD. Specialty pharmaceuticals for hyperlipidemia-impact on insurance premiums. N Engl J Med. 2015(17);373:1591-93.

6. Tóth PP, Potter D, Ming EE. Prevalence of lipid abnormalities in the United States: the National Health and Nutrition Examination Survey 2003-2006. J Clin Lipidol. 2012;6(4):325-30.

7. Carroll MD, Kit BK, Lacher DA, Shero ST, Mussolino ME. Trends in lipids and lipoproteins in US adults, 1988-2010. JAMA. 2012;308(15):1545-54.

8. Expert Panel on Detection, Evaluation, and Treatment of High Blood Cholesterol in Adults. Executive summary of the third report of the National Cholesterol Education Program (NCEP) Expert Panel on Detection, Evaluation, and Treatment of High Blood Cholesterol in Adults (Adult Treatment Panel III). JAMA. 2001;285(19):2486-97.

9. Pursnani A, Massaro JM, D'Agostino RB Sr, O’Donnell CJ, Hoffmann U. Guideline-based statin eligibility, coronary artery calcification, and cardiovascular events. JAMA. 2015;314(2):134-41.

10. Stone N, Robinson J, Lichtenstein AH, et al. 2013 ACC/AHA guideline on the treatment of blood cholesterol to reduce atherosclerotic cardiovascular risk in adults: a report of the American College of Cardiology /American Heart Association Task Force on Practice Guidelines. J Am Coll Cardiol. 2014;63(25 Pt B):2889-934.

11. Cannon CP, Blazing MA, Giugliano RP, et al; IMPROVE-IT Investigators. Ezetimibe added to statin therapy after acute coronary syndromes. N Engl J Med. 2015;372(25):2387-97.

12. Jacobson TA, Ito MK, Maki KC, et al. National Lipid Association recommendations for patient-centered management of dyslipidemia: Part 1—executive summary. J Clin Lipidol. 2014;8(5):473-88.

13. Shrank WH, Barlow JF, Brennan TA. New therapies in the treatment of high cholesterol: an argument to return to goal-based lipid guidelines. JAMA. 2015;314(14):1443-44.

14. Lopez D. Inhibition of PCSK9 as a novel strategy for the treatment of hypercholesterolemia. Drug News Perspect. 2008;21(6):323-30.

15. Zhang XL, Zhu QQ, Zhu L, et al. Safety and efficacy of anti-PCSK9 antibodies: a meta-analysis of 25 randomized, controlled trials. BMC Med. 2015;13:123

16. Sabatine MS, Giugliano RP, Wiviott SD, et al; Open-Label Study of LongTerm Evaluation against LDL Cholesterol (OSLER) Investigators. Efficacy and safety of evolocumab in reducing lipids and cardiovascular events. N Engl J Med. 2015;372(16):1500-09.

17. Navarese EP, Kolodziejczak M, Schulze V, et al. Effects of proprotein convertase subtilisin/kexin type 9 antibodies in adults with hypercholesterolemia: a systematic review and meta-analysis. Ann Intern Med. 2015;163(1):40-51. 
18. ClinicalTrials.gov. The evaluation of bococizumab (PF-04950615; RN316) in reducing the occurrence of major cardiovascular events in high risk subjects (SPIRE-2). Available at: https://clinicaltrials.gov/ct2/show/ NCT01975389. Accessed January 31, 2016.

19. ClinicalTrials.gov. ODYSSEY outcomes: evaluation of cardiovascular outcomes after an acute coronary syndrome during treatment with alirocumab SAR236553 (REGN727). Available at: https://clinicaltrials.gov/ ct2/show/NCT01663402. Accessed January 31, 2016.

20. ClinicalTrials.gov. Further cardiovascular outcomes research with pcsk9 inhibition in subjects with elevated risk (FOURIER). Available at: https:// clinicaltrials.gov/ct2/show/NCT01764633. Accessed January 31, 2016.

21. Husten L. Third strike for CETP inhibitors: Lilly halts big evacetrapib trial. CardioBrief. October 12, 2015. Available at: http://cardiobrief. org/2015/10/12/third-strike-for-cetp-inhibitors-lilly-halts-big-evacetrapibtrial/. Accessed January 31, 2016.

22. Hovingh GK, Ray KK, Boekholdt SM. Is cholesteryl ester transfer protein inhibition an effective strategy to reduce cardiovascular risk? CETP as a target to lower CVD risk: suspension of disbelief? Circulation. 2015;132(5):433-40.
23. Santilli J, Vogenberg FR. Key strategic trends that impact healthcare decision-making and stakeholder roles in the new marketplace. Am Health Drug Benefits. 2015;8(1):15-20. Available at: http://www.ncbi.nlm.nih.gov/ pmc/articles/PMC4415172/. Accessed January 31, 2016.

24. Centers for Medicare \& Medicaid Services. Announcement of Calendar Year (CY) 2015 Medicare Advantage Capitation Rates and Medicare Advantage and Part D Payment Policies and Final Call Letter. April 7, 2014. Available at: http://www.cms.gov/Medicare/Health-Plans/ MedicareAdvtgSpecRateStats/Downloads/Announcement2015.pdf. Accessed January 31, 2016.

25. Centers for Medicare \& Medicaid Services. Measures codes. Updated January 22, 2016. Available at: http://www.cms.gov/Medicare/QualityInitiatives-Patient-Assessment-Instruments/PQRS/MeasuresCodes.html. Accessed January 31, 2016

26. Chambers JD, Thorat T, Pyo J, Chenoweth M, Neumann PJ. Despite high costs, specialty drugs may offer value for money comparable to that of traditional drugs. Health Aff (Millwood). 2014;33(10):1751-60.

27. Institute for Clinical and Economic Review. PCSK9 inhibitors for treatment of high cholesterol: effectiveness, value, and value-based price benchmarks draft report. October 8, 2015. Available at: http://cepac.icer-review. org/wp-content/uploads/2015/04/PCSK9_REVISED_Draft_Report_100815. pdf. Accessed January 31, 2016. 